\title{
The Role of Enactment in Learning American Sign Language in Younger and Older Adults
}

\author{
Alison Fenney ${ }^{1}$ and Timothy D. Lee ${ }^{2}$ \\ ${ }^{1}$ McMaster Integrative Neuroscience Discovery and Study (MiNDS), McMaster University, ON, Canada L8S 4L8 \\ ${ }^{2}$ Kinesiology, McMaster University, ON, Canada L8S 4L8 \\ Correspondence should be addressed to Alison Fenney; fenneyal@mcmaster.ca
}

Received 18 August 2012; Accepted 16 September 2012

Academic Editors: K. Furukawa and D. G. Walker

Copyright (C) 2013 A. Fenney and T. D. Lee. This is an open access article distributed under the Creative Commons Attribution License, which permits unrestricted use, distribution, and reproduction in any medium, provided the original work is properly cited.

"Tell me, and I will forget. Show me, and I may remember. Involve me, and I will understand" (Confucius, 450 B.C). Philosophers
and scientists alike have pondered the question of the mind-body link for centuries. Recently the role of motor information has been
examined more specifically for a role in learning and memory. This paper describes a study using an errorless learning protocol
to teach characters to young and older persons in American Sign Language. Participants were assigned to one of two groups:
recognition (visually recognizing signs) or enactment (physically creating signs). Number of signs recalled and rate of forgetting
were compared between groups and across age cohorts. There were no significant differences, within either the younger or older
groups for number of items recalled. There were significant differences between recognition and enactment groups for rate of
forgetting, within young and old, suggesting that enactment improves the strength of memory for items learned, regardless of age.

\section{Introduction}

A growing volume of research is dedicated to understanding the role of physical engagement in learning, memory, and retrieval; however, this is by no means a new field of inquiry. Herman Ebbinghaus [1], a pioneer in the study of learning and memory, proposed a model that plotted the rate of forgetting and the factors that influenced the process of forgetting. Memory strength was dependent on two factors in his model: memory representation and active recall. Memory representation can be improved through environmental enrichment, which involved adding sight, sound, scent and tactile input to enhance the memory representation for an item. Active recall, or spaced repetition, improves memory strength by increasing time between recall so that the memory trace is being strengthened with each recall attempt $[2,3]$.

Environmental enrichment, the first component of Ebbinghaus's memory strength model, can include visual, auditory, olfactory, and tactile stimulation to engage multimodal processing. Enactment engages tactile and kinesthetic feedback by pairing a physical activity or movement to an item to be remembered. Research has consistently shown that enactment of verbal items provides enhanced memory performance compared to traditional verbal recall tasks [4]. An example of enactment of verbal items would be bringing your hand to your mouth to mime eating, when the spoon or fork is the verbal item to be remembered.

There are four main theories in the literature proposed to explain the enactment effect (also referred to as the subject performed task (SPT) effect). One theory, proposed by Cohen and Bean [5], suggests that the enactment effect is automatic and nonstrategic. Evidence exists that traditional strategic learning effects, found in verbal recall tasks, are not found when examining enactment [6]. Another theory $[7,8]$, suggests that the enactment effect is partially automatic and derives its benefit from the multimodal engagement that occurs during enactment, including visual, auditory, and tactile stimulation [6]. A third theory proposes that enactment encoding is organized into independent visual, auditory and motor programs, with the motoric being the most efficient, evidenced by the enactment effect [9]. Finally, a fourth theory contradicts the nonstrategic proposal of Cohen by suggesting that the enactment effect is entirely due 
to self-involvement and experiential registration, or episodic encoding of a personal experience [10].

The Cohen and Backman/Nilsson theories have converged in light of recent data. A revised view is that the nonstrategic nature of enactment is maintained with the specification of physical movement and multimodality encoding as critical components [6]. Several experiments have demonstrated an enactment effect, although the interpretation of the cause of this improvement remains controversial [11].

The second component of Ebbinghaus' model of memory is active recall. Active recall can improve memory strength through spacing, repetition, and timing. Manipulating the interval between study and recall, for example, can strengthen the memory trace. The spacing effect has been found across tasks and amongst varying age groups, as spacing information improves retention, compared to massed presentation, specifically in the long term [12]. The most effective form of spacing has been debated, with evidence supporting benefits of both equal and expanded spacing, with no reliable differences between the two $[13,14]$. Equal spacing refers to intervals of equal length (e.g., every interval is one minute), while expanded spacing involves increasing time intervals (e.g., the first interval is $30 \mathrm{sec}$, the next 1 minute, the next 2 minutes, and so on). Interestingly, expanded retrieval has proven effective in both young [15] and older adults with memory impairment $[16,17]$. A more recent study by Hochhalter et al. [18], involving persons with dementia, found comparable benefit for equal and expanded interval retrieval. It appears, regardless of the type of spacing, that there is a benefit to spacing of information and recall for learners across the lifespan.

Not only is the spacing of information and retrieval important, but so too is the type of experience and feedback during performance. The feedback of interest in this paper is the acknowledgement of correct or incorrect performance, referred to as trial and error (or errorless) learning. Trial and error involves learning in which attempts are made, errors are experienced, and more revised attempts are experienced (e.g., if that did not work maybe this will). In contrast, errorless learning requires the participant to have limited experience with error. This is achieved by observation of the correct performance and practice of that performance. Although in many cases trial and error may provide unique information by which to learn, this error must be minimized in some sense to limit the possible solutions to a proposed action [19]. Errorless learning was selected for this experiment so that it could be replicated in the future with clinical populations for comparison of this motor communication task across the lifespan. Errorless learning is thought to be beneficial for rehabilitation in persons with memory disorders as it reduces load on working memory [20]. Specifically, errorless learning has been found to enhance learning and memory, compared to errorful learning, in persons with amnesia [20].

The combined effect of enactment, expanded retrieval, and errorless learning for a motor communication task is not well understood. There is also a lack of evidence to accurately characterize the modulation of these effects across the lifespan. Evidence for improved roll of enactment and spaced retrieval in older, cognitively-impaired persons suggests that motor benefits may increase with greater cognitive challenge. This has implications for cognitive challenge and natural memory loss with aging.

The following studies were designed to leverage the benefits of environmental enrichment and active recall to improve memory. In the present study we examined the enactment effect, compared to recognition learning, using an expanded retrieval, errorless protocol in young (Experiment 1) and older persons (Experiment 2).

\section{Experiment 1}

The task selected for this experiment was learning of hand shapes for letters and words in American Sign Language (ASL). Sign language is a motor communication task that, for the learner, pairs the English word with the sign language hand-shape. This task was selected because it provided an opportunity for new learning (forming hand shapes) while contextually familiar (use of English letters, words) and involved physical engagement. Expanded retrieval was used for recall of signs to test this schedule's intrinsic ability to improve memory, and examine the combination of enactment and expanded retrieval. An errorless learning protocol was selected to examine the characteristics of learning this motor task in absence of error experience. We hypothesized that enactment would improve learning, memory and retention of learned items, and result in better overall performance in comparison to merely learning to recognize items.

2.1. Participants. Twenty participants, aged $18-30$ (eight males and 12 females), were recruited from McMaster University's graduate and undergraduate population. All participants provided informed consent, approved by the McMaster University Human Research Ethics Board. Exclusion criteria involved any prior experience with sign language, collected via self-report.

2.2. Method. Participants were assigned to one of two groups: enactment or recognition. Each group viewed an expert performing a sequence of hand shapes and were asked to review this video with the aim of (a) recognizing the sign in a future viewing (recognition group) or (b) enacting the sign in a future cued scenario (enactment group). Hand shapes represented letters and words in ASL, and were presented in groups of 3-6. Hand shapes increased in difficulty and number per set as the experiment advanced in order to maintain an increasing level of challenge for participants.

The recognition group was encouraged to watch the video multiple times to gain familiarity with the signs. The name of each sign was written in English at the top of the video. Participants were instructed not to attempt to form the signs with their hands. During recall, after the initial viewing, participants in this group viewed the same video of expert performance without the names of each sign written on the slide.

The enactment group was encouraged to create hand shapes along with the expert viewed on the video at the beginning of each set. The video showed a close up view of 


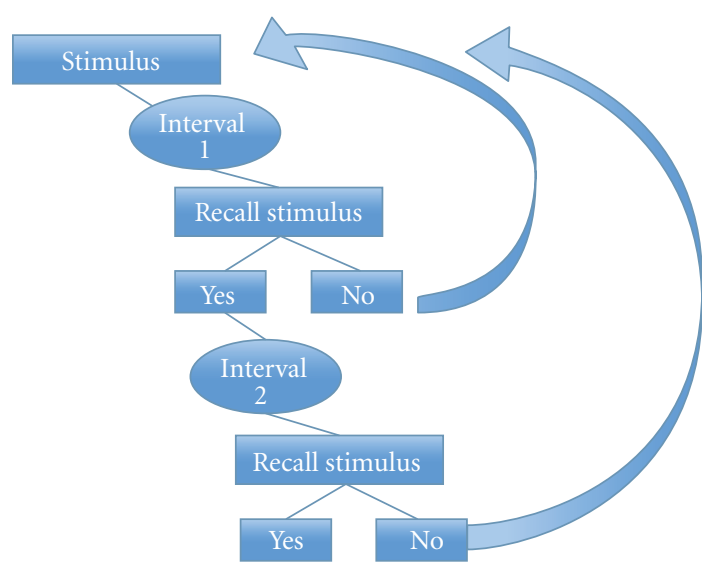

Figure 1: Errorless Learning Protocol.

the expert, torso and face, completing hand-shapes in ASL. After each recall interval participants were presented with the letters or words in English and asked to form the ASL hand shape for those items. All trials were filmed to ensure proper formation of hand shapes.

Time intervals between recall were expanding, $10 \mathrm{~s}$, $20 \mathrm{~s} \cdots 160 \mathrm{~s}$ (see Figure 1). During intervals participants were engaged in one of three computer games: blackjack, hi-lo, or a shell game. The interference in the computer game was nonmotor; participants instructed the experimenter on which moves, selections they wanted to make.

If the participant had successfully recalled items from the first to the last time point (from 30 seconds, all the way to 160 seconds), they moved on to the next set of signs. If an error was made they returned to the instructional slide and began the interval sequence again. Once the second set of signs was completed participants were asked to recall the signs from the previous set. If they were unable to recall the signs in the previous set the experiment ended. The experiment was halted at this point on the assumption that if the participant was unable to remember the previous set of signs, that they had reached their short-term memory capacity, and could retain no new information without replacing the older information.

Participants were asked to perform an immediate recall test of all signs they had practiced (in the same order) and a random recall test (items were presented in a random order), if participants failed to recall a sign in sequence the test was discontinued. Participants returned six to eight weeks later for a retention test, in which they were asked to recognize signs from the expert video, as well as enact signs when prompted with the English word. All participants performed both retention tests; therefore each group had one familiar recall and one unfamiliar (based on their original learning condition).

Dependent Measures. Number of items recalled was compared within group across retention sessions as well as between groups. There were three data points, immediate recall, random recall, and retention. Immediate recall was the number of items recalled by participants during the learning session. Random recall was the recall for items in a order different from that of learning. The final retention test occurred six weeks after the initial learning session. Participants were required to recall items by recognizing them in the expert video, or performing hand-shapes when prompted by the English word. A total of thirty-two letters and words were presented to participants in this experiment. At the final retention test participants were asked to recall half the items via recognition of expert performance, and the other half through enactment of signs when an English word was presented. This created a familiar recall (recall in the method engaged during learning) and an unfamiliar recall (recall in a different method then engaged during learning).

Rate of forgetting was calculated by subtracting retention from immediate recall, dividing by immediate recall and multiplying by 100 to calculate a percentage. Rate of forgetting represented the proportion of signs learned that were recalled at the retention period. This measure allows for comparison between participants and groups with variable number of items recalled. Rate of forgetting represents the strength of memory for items.

2.3. Results. A 2-factor between/within ANOVA was used to analyze number of items recalled between and within groups. The values used in the ANOVA were immediate recall and final retention test (after 6 weeks) for the recognition and enactment groups. There were no significant differences between the recognition and enactment groups. Comparison of condition, in immediate recall and retention, revealed a significant difference $(F(1,36)=254.18, P<0.01)$ with the retention test eliciting a significantly lower number of items remembered than the recall test (i.e., there were a significant number of items participants "forgot" during the retention period). Rate of forgetting was compared between groups using a $t$-test to determine if enactment had a greater or lesser rate of forgetting compared to recognition; differences reached significance $(t(18)=1.848, P<0.05,1$-tailed $)$. The recognition group had a significantly greater rate of forgetting then the enactment group (see Figure 2-Table 1 for group means and standard deviations). There was no significant difference in items recalled in the familiar condition between the enactment $(M=5.8)$ and recognition groups $(M=$ 5.18), $(t(18)=0.56, P=0.577)$. This finding suggests that encoding through enactment or recognition provided equivalent benefit at recall when the items were recalled in a familiar method (enactment of signs for the enactment group, and recognition of signs for the recognition group).

There was a significant difference in items recalled in the unfamiliar condition between enactment $(M=6.55)$ and recognition groups $(M=2.91),(t=2.65, P=0.0164)$. This finding suggests that encoding through enactment provided a greater benefit at recall for items in an unfamiliar context, compared to recognition. The enactment group was better at recognizing signs performed by an expert, compared to the recognition group's ability to enact signs at recall.

2.4. Discussion. These results suggest that for younger people there is no specific benefit of enactment in learning, compared to recognition, for the number of items recalled. The 


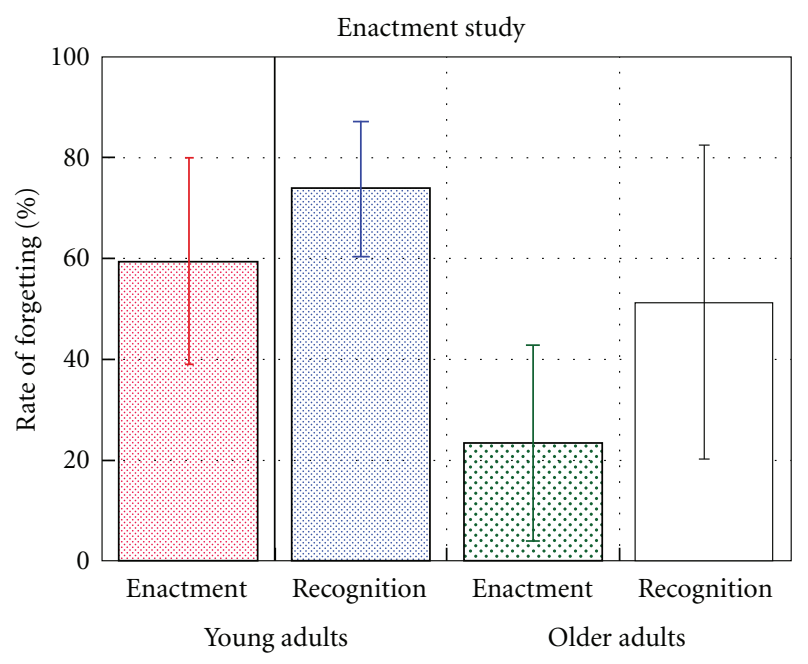

FIGURE 2: Rate of forgetting for both younger and older adults.

TABLE 1: Group means for both older and younger participants, for recall comparisons.

\begin{tabular}{lcccc}
\hline & \multicolumn{2}{c}{ Young } & \multicolumn{2}{c}{ Older } \\
& Mean & SD & Mean & SD \\
\hline Enactment & & & & \\
$\quad$ Recall & 31.11 & 1.83 & 10.88 & 10.37 \\
Random & 22.11 & 10.04 & 11.50 & 10.85 \\
Retention 1 wk. & - & - & 8.75 & 6.88 \\
Retention 3 wks. & - & - & 8.50 & 5.66 \\
Retention 6 wks. & 12.44 & 5.90 & - & - \\
Rate of forgetting & 59.00 & 20.62 & 2.38 & 5.58 \\
\hline Recognition & & & & \\
Recall & 30.55 & 3.21 & 15.57 & 6.24 \\
Random & 19.09 & 14.65 & 13.43 & 7.14 \\
Retention 1 wk. & - & - & 8.57 & 5.47 \\
Retention 3 wks. & - & - & 6.71 & 3.90 \\
Retention 6 wks. & 8.09 & 4.30 & - & - \\
Rate of forgetting & 73.48 & 13.42 & 8.86 & 7.58 \\
\hline
\end{tabular}

ceiling effect has been found previously in younger adults in enactment encoding conditions [21], but this has not been in direct comparison to recognition. This lack of difference may be a result of insufficient challenge at the initial learning stage, or redundancy of sensory information from visual observation and physical engagement. The significant difference in rate of forgetting suggests that benefits of enactment may exist in the strength of the memory for items learned. Participants in the enactment group recalled a greater proportion of signs at the 6-week retention test, demonstrating a better memory for items than those in the recognition group.

The lack of enactment effect in number of items recalled in the younger group may be a result of several factors: a ceiling effect, errorless protocol at recall, or nature of the task. There was a ceiling effect during learning, with most participants recalling all items during the acquisition period (a total of 32). Another possible explanation may be the errorless format of the recall sessions. The random recall session was ended immediately if a participant was unable to recall a sign, but this limited our ability to capture any signs later in the order of recall. Both groups experienced a significant decrease in number of items recalled at retention, demonstrating significant rates of forgetting. This decrease could be a result of poor encoding at learning and initial recall, or due to the length of the retention period, six weeks. The nature of the task, motor communication, may have been very intuitive to participants, and not provided the level of challenge required to engage the enactment effect. Comparison of familiar versus unfamiliar retention scores revealed that the enactment group demonstrated significantly better retention scores in the unfamiliar retention test. This finding is in contradiction to theories such as encoding specificity [22], and suggests an improved strength of memory with enactment encoding.

Limitations. An errorless protocol was extended into the random recall portion of the experiment. Immediate recall was used for comparison because this may have artificially reduced the number of items recalled by a participant. If a participant had difficulty remembering a particular sign they were prevented from recalling any other signs that occurred later in the recall order. As a result, the errorless protocol was not applied in experiment two. The length of the retention period may have contributed to lack of effect, and therefore, was adjusted to capture a more accurate picture of recall in the older cohort.

\section{Experiment 2}

In Experiment 2 the hypothesis remained the same, that enactment would improve learning, memory and retention of learned items, and garner better overall performance in comparison to merely learning to recognize items. If errorless learning can be beneficial when explicit learning, or working memory, is challenged $[20,23]$, then enactment paired with errorless learning should improve performance in older adults compared to a recognition protocol.

3.1. Participants. All participants were recruited using advertisements in an online news outlet (the McMaster University Daily News) and posters on campus. All participants provided informed consent. Fifteen participants aged 54-77, ten females and five males, were tested. Groups were pseudobalanced with the goal of equating for gender and age. Exclusion criteria involved any prior experience with sign language, collected via self-report.

3.2. Method. All participants were briefed about the format of the experiment prior to beginning. The experimenter administered the mini-mental state exam to each participant, and the data were used for within-cohort comparison only. Participants were assigned to one of two groups: recognition or enactment. The protocol of this experiment was identical to that of experiment one, up to the point of the random recall component. For this experiment, during the random recall 
test, participants were able to "skip" a sign they could not recall and continue the test.

The spacing and number of retention tests were also adjusted for this experiment. Participants returned one week and two weeks after the initial session for retention testing. At the final retention test participants were asked to recall half the items via recognition of expert performance, and the other half through enactment of signs when the English word was presented. This created a familiar recall (recall in the method engaged during learning) and an unfamiliar recall (recall in a different method then engaged during learning).

Two measures were used to ensure homogeneity of groups in the older cohort: a mini-mental state exam (MMSE) and a tactile discrimination task. The MMSE was included to ensure that participants did not suffer from any significant memory impairment prior to participation. The purpose of the tactile discrimination task was to capture the motor ability to discriminate between nonsense shapes and familiar letter shapes. This was included to parse out the ability to link motor information to meaning, letters or language, or to nonsense shapes, or physical information lacking a specific context.

The participants were asked to complete a letter and nonsense shape tactile discrimination task [24]. Participants were blindfolded and asked to "feel" a shape with the right hand. The blindfold was removed and the participant was asked to select the shape they had felt from a screen containing 6 letters and/or shapes. The test was repeated with the left hand. There were a total of 8 shapes and letters.

3.3. Results. The mean age of participants participating in this study was 67.93 years old, with a standard deviation of 7.56 years. A $t$-test was used to compare MMSE and tactile discrimination task scores between groups. There were no significant differences for MMSE score $(M=28.69, \mathrm{SD}=$ $1.4, t(12)=-1.072, P<0.05)$. There were no significant differences in tactile discrimination in the left hand $(M=$ $6.78, \mathrm{SD}=1, t(11)=-0.746, P<0.05)$; however, differences reached significance in the right hand $(M=6.08, \mathrm{SD}=1.5$, $t(11)=3.187, P>0.05)$. The enactment group $(M=$ 7) made a greater mean number of correct discriminations compared to the recognition group $(M=5)$.

A 2-factor ANOVA was used to analyze within and between group differences. There were no significant differences between the recognition and enactment groups $(F(1,26)=0.265, P>0.05)$. There was a main effect of condition, immediate recall and retention, $(F(1,26)=$ 4.611, $P<0.05)$. Rate of forgetting was $23 \%$ and $51 \%$ for the enactment and recognition groups, respectively. The difference between rates of forgetting was significant $(t(13)=$ 2.128, $P<0.05)$, with the recognition group having a higher rate of forgetting when compared to the enactment group (see Table 1 for group means and standard deviations). There was no significant difference in items recalled in the familiar condition between the enactment $(M=4.25)$ and recognition groups $(M=3.57),(t(10)=0.72, P=$ $0.49)$ or the unfamiliar condition, enactment $(M=4.75)$ and recognition $(M=2.43),(t(10)=1.47, P=0.17)$. This finding suggests that encoding through enactment or recognition provided equivalent benefit at recall when the items were recalled in a familiar method (enactment of signs for the enactment group, and recognition of signs for the recognition group).

3.4. Discussion. MMSE data revealed no significant differences in cognitive ability between groups for the older adults. Analysis of tactile discrimination task results revealed that all participants had the capacity to learn to discriminate between language associated and non-sense shapes. There was a significant difference in the number of correct discriminations in the right hand between groups, with the enactment group having a greater number of correct discriminations in the right hand compared to the recognition group. This may be explained by differences in handedness.

Although there was a trend towards enactment improving performance at retention in the older group, the betweengroup difference failed to reach significance for overall number of items recalled. Differences in condition illustrate the significant number of items forgotten between recall sessions. Rate of forgetting was significantly different between groups, with the recognition group having a greater rate of forgetting, remembering a lower proportion of signs at retention compared to the enactment group. Individuals in the recognition group recalled more items during training and at immediate recall, but had a greater rate of forgetting. Individuals in the enactment group did not learn or recall as many items at training and immediate recall but retained a greater proportion of what they did learn at retention. This finding suggests that the benefit of enactment is not in volume of items learned but the strength of memory for those items. The strength of memory, in the case of older adults, improved overall number of items recalled but did not significantly impact performance in familiar or unfamiliar recall.

\section{Comparing Experiment 1 and 2 Results}

In order to understand how the effect of enactment may be modulated by age a comparison was completed between experiment one and two. The difference between protocols experienced by the younger and older cohorts included the difference in retention period (6-8 weeks for the younger adults, and 3 weeks for the older adults), and the use of errorless learning at randomized recall (used for the first half of younger adults but removed for the second half of younger adults, and the older adults). The randomized recall score was not used for comparison within or between groups or cohorts. If the reduction in retention period influenced this comparison, we would assume that overall number of items recalled would be higher for the older adults (with shorter retention period) than young adults, but this was not found. The finding suggests that comparison between the two experiments is appropriate, even given the differences in retention period.

A three-factor ANOVA was used to compare the results of the first and second experiments. There was a significant effect of age for number of items recalled $(F(1,62)=57.02$, $P<0.05)$, condition $(F(1,62)=94.29, P<0.05)$, and $\mathrm{a}$ 
significant interaction between age and condition $(F(1,62)=$ $30.79, P<0.05)$. Holm-Sidak post hoc analysis of this interaction, revealed a significant difference for age within enactment, with older adults performing better relative to young $(t(1)=6.519, P<0.05)$, and age within recognition, with younger performing better then older adults $(t(1)=7.198$, $P<0.05)$ (see Figure 2).

A post hoc power calculation was done to help determine the accuracy of statistical outcomes for the comparison of enactment and recognition groups for younger $(d=0.9)$ and older adults $(d=0.64)$. The Cohen's $d$ value for both the young and older adults suggests that the statistical result carries practical significance (i.e., decreased risk of type II error was made, [25]).

\section{General Discussion}

These results support a beneficial role for enactment in learning and recall [11]. This beneficial role may be due to the engagement by the subject in performance of task, as well as the observation of the experimenter completing the task, both of which have been found to produce an enactment effect [26]. There are several limitations to analysis between these two experiments, namely the ceiling effect of performance in young at initial learning and the alterations in the retention protocol for the older adults. Keeping in mind these challenges we compared the two groups in an attempt to characterize the differences in the way each age group utilized motor information in the form of enactment. Both young and older adults experienced a reduced rate of forgetting with enactment, improving strength of memory for items compared to the recognition group. There were significant differences in initial learning, clearly identifying a capacity difference in young versus older adults. The apparent increased benefit from enactment for number of items recalled with age may be related to cognitive ability or differences in memory capacity that can be challenged with age. It has been suggested that multisensory input can be highly redundant [27]. Therefore, when all systems are supposedly operating at their best, such as in young cognitively healthy adults, there is no added benefit from motor information. For the older learners, all systems may be challenged, with cognitive or short-term memory impairment; this may be offset by integration of motor information to strengthen memory. This interaction suggests that motor information may provide compensatory information to bolster memory systems challenged by age related declines in performance.

\section{Conclusion}

Results from these experiments show an apparent age effect of enactment, with young experiencing no added benefit of enactment across learning or retention, and older participants nearing a significant improvement with enactment. This suggests a benefit of the use of motor information encoding during enactment in the presence of cognitive or short-term memory challenge. Further research should focus on examining this effect in persons with memory impairment, specifically dementia. Utilizing this protocol with persons with dementia will add to the enactment literature in this population, and may reveal a valid therapeutic tool for communication later in the disease. Using a motor communication task may increase the quality and quantity of communication for persons with word finding and verbal memory impairments, improving quality of life.

\section{References}

[1] H. Ebbinghaus, Memory: A Contribution to experimental Psychology, Dover, Mineola, NY, USA, 1964.

[2] J. D. Karpicke and J. R. Blunt, "Retrieval practice produces more learning than elaborative studying with concept mapping," Science, vol. 331, no. 6018, pp. 772-775, 2011.

[3] N. Kornell, A. D. Castel, T. S. Eich, and R. A. Bjork, "Spacing as the friend of both memory and induction in young and older adults," Psychology and Aging, vol. 25, no. 2, pp. 498-503, 2010.

[4] J. D. Von Essen and L. G. Nilsson, "Memory effects of motor activation in subject-performed tasks and sign language," Psychonomic Bulletin and Review, vol. 10, no. 2, pp. 445-449, 2003.

[5] R. L. Cohen and G. Bean, "Memory in educable mentally retarded adults: Deficit in subject or experimenter?" Intelligence, vol. 7, no. 3, pp. 287-298, 1983.

[6] L. Nilsson, "Remembering Actions and Words," in Oxford Handbook of Memory, E. Tulving and F. I. M. Craik, Eds., pp. 137-148, Oxford Press, New York. NY, USA, 2000.

[7] L. Bäckman and L. G. Nilsson, "Aging effects in free recall: an exception to the rule," Human Learning, vol. 3, pp. 53-69, 1984.

[8] L. Backman and L. G. Nilsson, "Prerequisites for lack of age differences in memory performance," Experimental Aging Research, vol. 11, no. 2, pp. 67-73, 1985.

[9] E. Tulving and F. I. M. Craik, Eds., The Oxford Handbook of Memory, Oxford University Press, Oxford, UK, 2005.

[10] R. Kormi-Nouri, "The nature of memory for action events: an episodic integration view," European Journal of Cognitive Psychology, vol. 7, pp. 337-363, 1995.

[11] P. Feyereisen, "Enactment effects and integration processes in younger and older adults' memory for actions," Memory, vol. 17, no. 4, pp. 374-385, 2009.

[12] D. A. Balota, J. Duchek, and J. Logan, "Is expanded retrieval practice a superior form of spaced retrieval?" in The Foundations of Remembering, J. S. Nairne, Ed., Psychology Press, Florence, Ky, USA, 2007.

[13] D. A. Balota, J. M. Duchek, S. D. Sergent-Marshall, and H. L. Roediger, "Does expanded retrieval produce benefits over equal-interval spacing? Explorations of spacing effects in healthy aging and early stage Alzheimer's disease," Psychology and Aging, vol. 21, no. 1, pp. 19-31, 2006.

[14] J. M. Logan and D. A. Balota, "Expanded vs. equal interval spaced retrieval practice: Exploring different schedules of spacing and retention interval in younger and older adults," Aging, Neuropsychology, and Cognition, vol. 15, no. 3, pp. 257-280, 2008.

[15] C. P. Rea and V. Modigliani, "The spacing effect in 4- to 9-yearold children," Memory and Cognition, vol. 15, no. 5, pp. 436-443, 1987.

[16] C. J. Camp, J. W. Foss, A. M. O’Hanlon, and A. B. Stevens, "Memory interventions for persons with dementia," Applied Cognitive Psychology, vol. 10, no. 3, pp. 193-210, 1996. 
[17] C. J. Camp and M. J. Bird, "Retrieval strategies as a rehabilitation aid for cognitive loss in pathological aging," in Cognitive Rehabilitation in Old Age, R. D. Hill, L. Backman, and A. S. Neely, Eds., pp. 224-228, Oxford University Press, New York, NY, USA, 2000.

[18] A. K. Hochhalter, J. B. Overmier, S. M. Gasper, B. L. Bakke, and R. J. Holub, "A comparison of spaced retrieval to other schedules of practice for people with dementia," Experimental Aging Research, vol. 31, no. 2, pp. 101-118, 2005.

[19] L. Fetters, "Perspective on variability in the development of human action," Physical Therapy, vol. 90, no. 12, pp. 1860-1867, 2010.

[20] A. Baddeley and B. A. Wilson, "When implicit learning fails: Amnesia and the problem of error elimination," Neuropsychologia, vol. 32, no. 1, pp. 53-68, 1994.

[21] M. Lövdén, M. Rönnlund, and L. G. Nilsson, "Remembering and knowing in adulthood: Effects of enacted encoding and relations to processing speed," Aging, Neuropsychology, and Cognition, vol. 9, no. 3, pp. 184-200, 2002.

[22] E. Tulving and D. M. Thomson, "Encoding specificity and retrieval processes in episodic memory," PSYCHOL.REV., vol. 80, no. 5, pp. 352-373, 1973.

[23] D. C. Park and P. Reuter-Lorenz, "The adaptive brain: Aging and neurocognitive scaffolding," Annual Review of Psychology, vol. 60, pp. 173-196, 2009.

[24] S. F. Witelson, "Hemispheric specialization for linguistic and nonlinguistic tactual perception using a dichotomous stimulation technique," Cortex, vol. 10, no. 1, pp. 3-17, 1974.

[25] F. M. Wolf, Meta-Analysis: Quantitative Methods for Research Synthesis, Sage, Beverly Hills, Calif, USA, 1986.

[26] N. W. Mulligan and S. L. Hornstein, "Memory for actions: Self-performed tasks and the reenactment effect," Memory and Cognition, vol. 31, no. 3, pp. 412-421, 2003.

[27] J. Jeka, K. S. Oie, and T. Kiemel, "Multisensory information for human postural control: Integrating touch and vision," Experimental Brain Research, vol. 134, no. 1, pp. 107-125, 2000. 


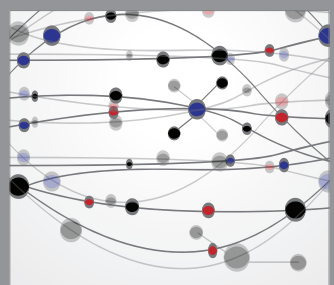

The Scientific World Journal
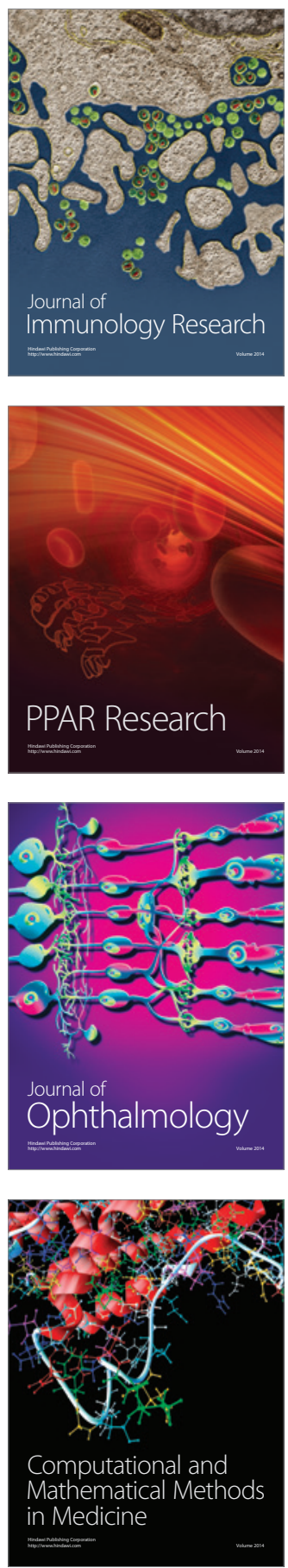

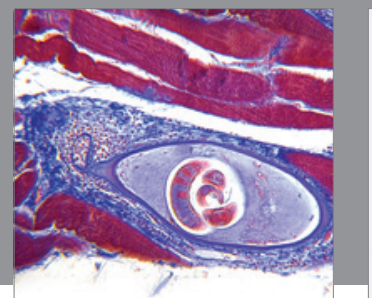

Gastroenterology

Research and Practice
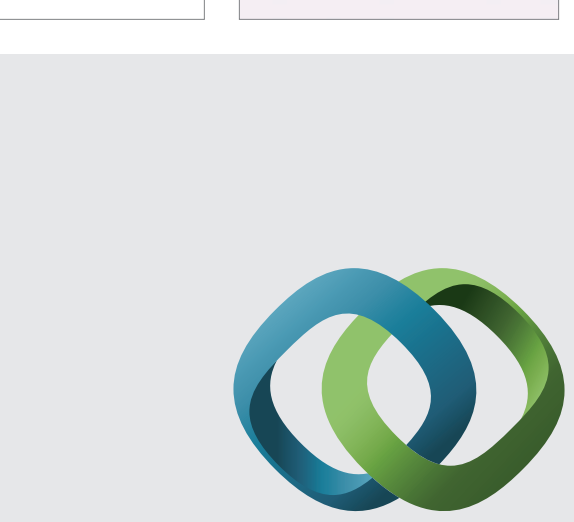

\section{Hindawi}

Submit your manuscripts at

http://www.hindawi.com
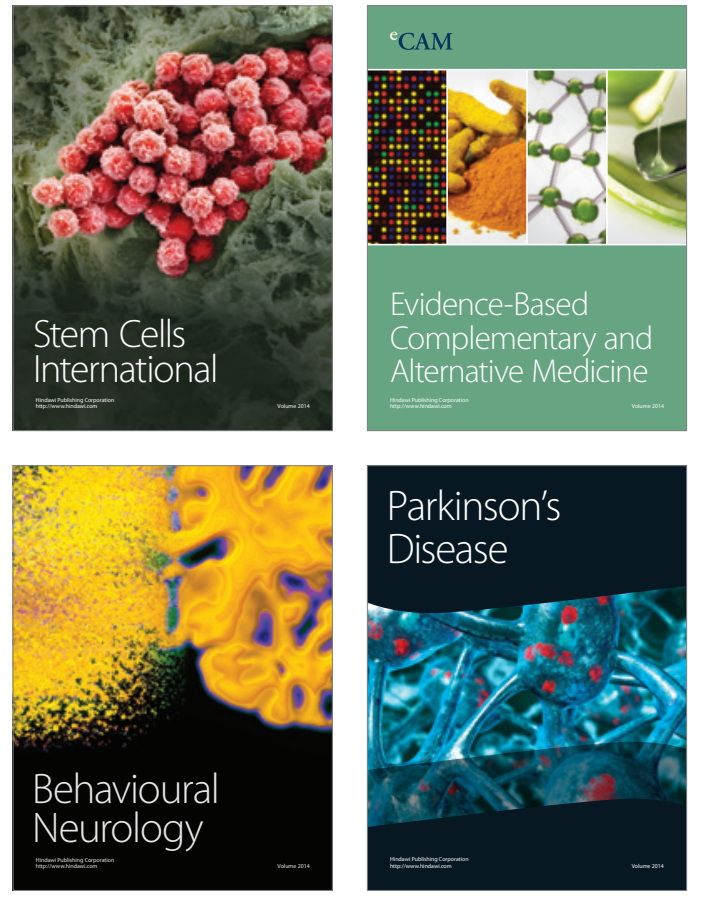
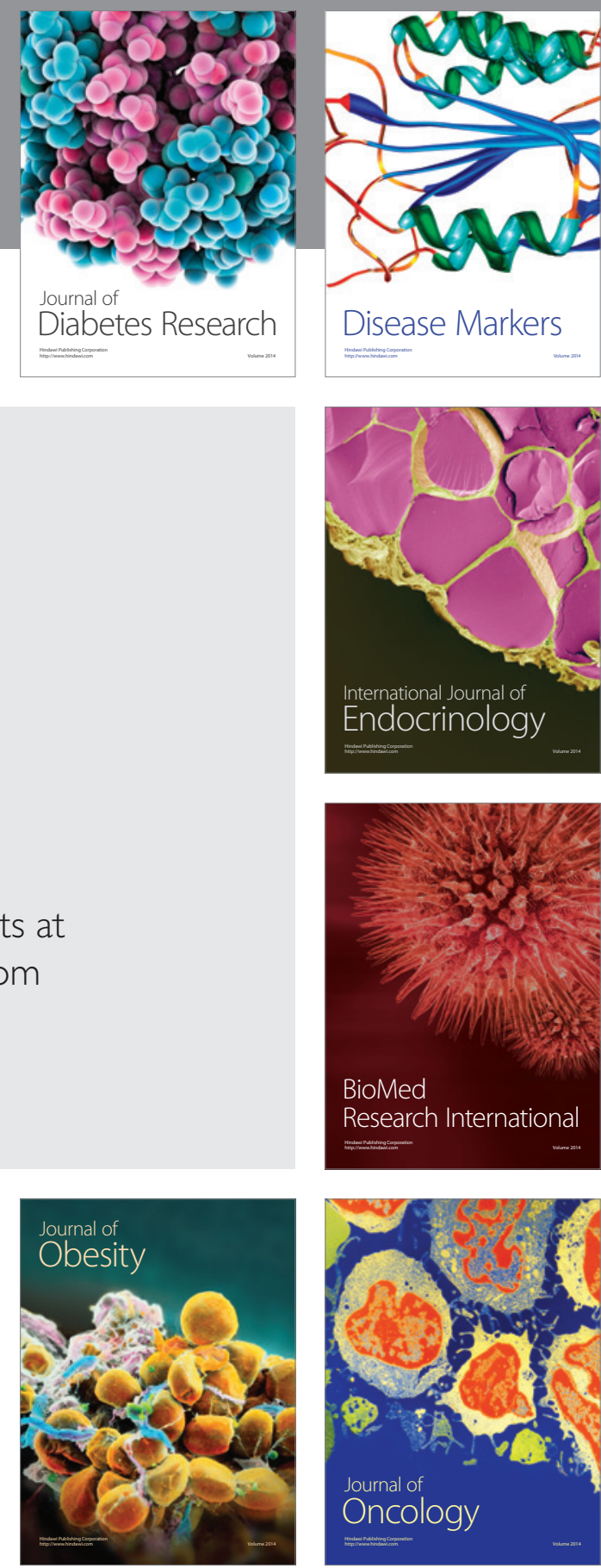

Disease Markers
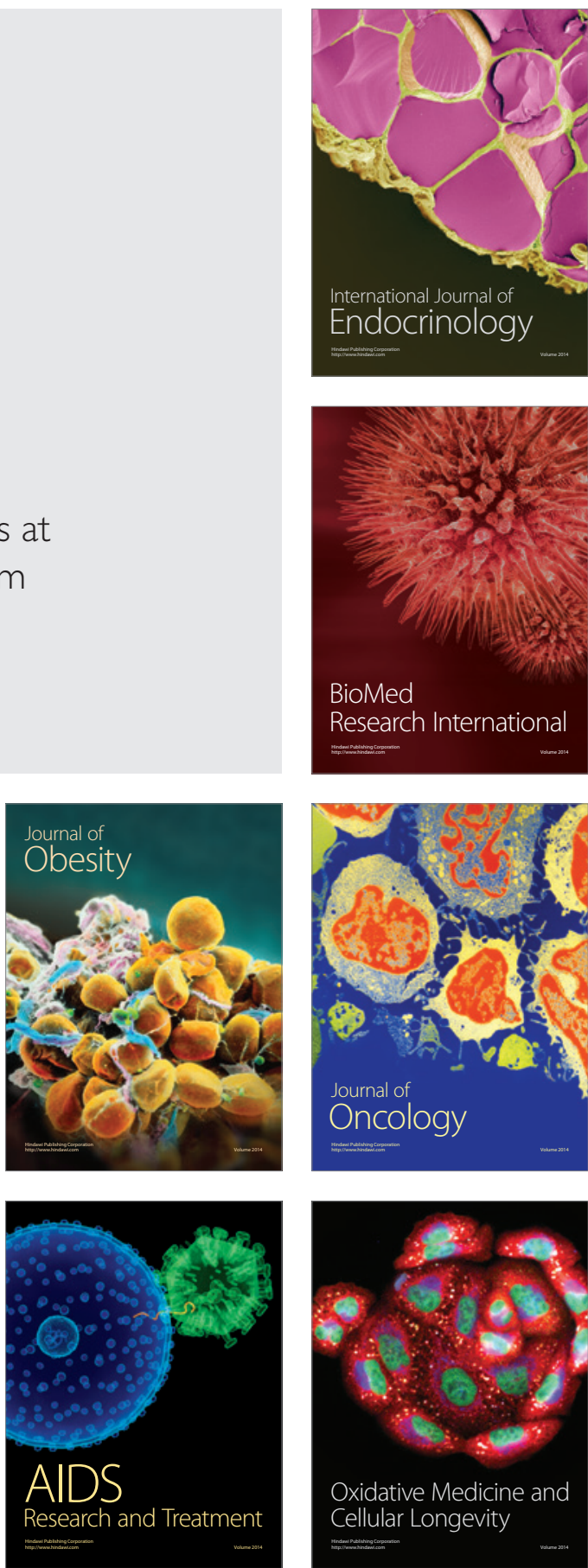